Cite this: RSC Adv., 2014, 4, 16250

Received 6th March 2014

Accepted 24th March 2014

DOI: $10.1039 / c 4 r a 01961 j$

www.rsc.org/advances

\section{PE/PEO blends compatibilized by PE brush immobilized on MWNTs: improved interfacial and structural properties}

\author{
Prasanna Kumar S. Mural, ${ }^{a}$ Manish Singh Rana, ${ }^{b}$ Giridhar Madras ${ }^{a b}$ \\ and Suryasarathi Bose*c
}

Polyolefin based blends have tremendous commercial importance in view of their exceptional properties. In this study the interface of a biphasic polymer blend of PE (polyethylene) and PEO (polyethylene oxide) has been tailored to reduce the interfacial tension between the phases and to render finer morphology. This was accomplished by employing various strategies like addition of maleated PE (PE grafted maleic anhydride), immobilizing PE chains, ex situ, onto MWNTs by covalent grafting, and in situ grafting of PE chains onto MWNTs during melt processing. Multiwalled nanotubes (MWNTs) with different surface functional groups have been synthesized either a priori or were facilitated during melt mixing at higher temperature. $\mathrm{NH}_{2}$ terminated MWNTs were synthesized by grafting ethylene diamine (EDA) onto carboxyl functionalized carbon nanotubes ( $\mathrm{COOH}-\mathrm{MWNTs})$ and further, was used to reactively couple with maleated PE to immobilize PE chains on the surface of MWNTs. The covalent coupling of maleated PE with $\mathrm{NH}_{2}$ terminated MWNTs was also realized in situ in the melt extruder at high temperature. Both $\mathrm{NH}_{2}$ terminated MWNTs and the in situ formed PE brush on MWNTs during melt mixing, revealed a significant improvement in the mechanical properties of the blend besides remarkably improving the dispersion of the minor phase (PEO) in the blends. Structural properties of the composites were evaluated and the tensile fractured morphology was assessed using scanning electron microscopy.

\section{Introduction}

Blending of polymers is a versatile tool to achieve targeted properties with the existing polymers. ${ }^{1,2}$ In general, most polymeric blends are often thermodynamically immiscible in nature due to a small gain in entropy on mixing. The final morphology of the blends is contingent on the composition, viscosity ratio, interfacial tension, processing etc., resulting in a wide variety of microstructures such as matrix-droplet, fibrillar, lamellar or continuous. A compatibilizer like a block or a graft co-polymer is often added to reduce the interfacial tension between the constituents and to generate a finer morphology. ${ }^{3-5}$ For instance, Hashmi et al., ${ }^{6}$ showed that the addition of styrene-butadiene-styrene (SBS) copolymer to polypropylene/polystyrene blends exhibited a finer morphology with respect to blends without SBS. Meltzer et al.,7 observed that addition of maleated PE to PE/PEO blend led to a significant refinement in morphology due to

${ }^{a}$ Center for Nano Science and Engineering, Indian Institute of Science, Bangalore-560012, India

${ }^{b}$ Department of Chemical Engineering, Indian Institute of Science, Bangalore-560012, India

'Department of Materials Engineering, Indian Institute of Science, Bangalore-560012, India. E-mail: sbose@materials.iisc.ernet.in; Tel: +91-80-22933407 reactive compatibilization between maleic anhydride (MAH) and the hydroxyl ends of PEO.

Polyolefins represent a class of important commodity polymers because of its good processability, low cost and good chemical resistance and hence, has tremendous commercial importance. Current research focuses to develop polyolefin based new materials with desired performance in view of its commercial importance. However, due to inherent immiscibility with other macromolecules, the desired properties are often not realized by mere blending. Further, the nonpolar nature of the polyolefins add to the challenge of compatibilizing polyolefinic blends. Therefore, various methods have been explored in the recent past to compatibilize polyolefinic blends.

The different morphologies generated during melt processing of polymer blends can be tailored to explore various strategic applications. Among the different morphologies, the matrix-droplet type offer a unique route to design substrates for separation technology. Polymeric membranes, in general, are derived from phase inversion process, thermally induced phase separation (TIPS), track etching or by solvent free methods like stretching melt-cast polymer films etc. While these methods have their own merits and demerits, an alternative route in designing polymer based membranes, which has recently attracted lot of attention, is by selectively etching one of the phases from a bi-phasic blend. ${ }^{7,8}$ 
Recent studies report the key role of nanoparticles (NPs) in compatibilizing binary blends. However, the underlying mechanism is significantly different from the classical approach (i.e. block/graft copolymer) of compatibilizing binary blends. Many factors such as surface free energy of the component, melt viscosity, processing parameters etc. govern the localization of NPs in a binary blend. Besides rendering finer microstructures, the NPs also offer unique functional properties which make them a potential candidate in designing polymer based nanocomposites. Among the NPs, carbon nanotubes (CNTs) have attracted a great deal of interest owing to their exceptional properties. While the majority of the work reported till date focuses on the electrical, mechanical and thermal properties of the composites, their key role in stabilizing binary blends has also been realized recently.

Therefore, in this study, we have determined the mechanical properties and dispersion of the phases in the presence of functionalized multiwalled nanotubes (MWNTs). The mechanical properties and the morphology of the blends were determined. We have attempted to reduce the interfacial tension between the phases by employing different strategies. These include the addition of PE grafted maleic anhydride, ex situ immobilizing PE chains on to MWNTs and in situ grafting of PE chains on to amine functionalized MWNTs. We have compared the various strategies and determined the blend that exhibits enhanced mechanical properties and shows increased dispersion of PEO in PE. The reasons for these observations are also discussed in detail.

\section{Experimental}

\section{Materials}

Low density polyethylene (PE) (melt flow index of $25 \mathrm{~g}$ per 10 min and density of $0.925 \mathrm{~g} \mathrm{~cm}^{-3}$ ), polyethylene oxide (PEO, $M_{\mathrm{v}}=400000$ ), maleated polyethylene (PE grafted with maleic anhydride), and ethylene diamine (EDA) were obtained from Sigma Aldrich. The pristine MWNTs (p-MWNTs, NC 7000 of 90\% purity and $1.5 \mu \mathrm{m}$ long) and acid functionalized MWNTs (COOH-MWNTs, NC 3150 of 95\% purity and $<1 \mu \mathrm{m}$ long) were obtained from Nanocyl, Belgium. Solvents like tetrahydrafuran (THF), xylene, and thionyl chloride $\left(\mathrm{SOCl}_{2}\right)$ were obtained from Merck, India and were used without further purification.

\section{Synthesis of $\mathrm{NH}_{2}$ terminated MWNTs}

$\mathrm{NH}_{2}$ terminated MWNTs were synthesized from carboxyl $(-\mathrm{COOH})$ functionalized MWNTs using EDA; a convenient method that has been widely applied in functionalizing MWNTs. Typically, $800 \mathrm{mg}$ of MWNT-COOH was suspended in THF and was mixed with $80 \mathrm{~mL}$ of $\mathrm{SOCl}_{2}$ in a $250 \mathrm{~mL}$ round bottomed flask. The mixture was then stirred at $80^{\circ} \mathrm{C}$ for $24 \mathrm{~h}$ in presence of EDA. The powder was isolated by vacuum filtration and was washed with anhydrous THF to remove excess $\mathrm{SOCl}_{2}$. This purification cycle was repeated couple of times to ensure complete removal of excess $\mathrm{SOCl}_{2}$ and the final product was vacuum dried for $24 \mathrm{~h}$ at $80{ }^{\circ} \mathrm{C}$.

\section{Immobilizing PE on the surface of MWNTs}

PE grafted MWNT were prepared by grafting maleated PE on to $\mathrm{NH}_{2}$ terminated MWNTs via an amidation reaction between the amine groups of $\mathrm{NH}_{2}$ terminated MWNTs and the maleic anhydride groups of maleated PE. In a typical reaction, $50 \mathrm{mg}$ of $\mathrm{NH}_{2}$ terminated MWNTs was initially suspended in $10 \mathrm{~mL}$ of xylene. The mixture was sonicated for half an hour to exfoliate the nanotubes. $120 \mathrm{mg}$ of maleated $\mathrm{PE}$ was then added to this mixture and was refluxed at $100{ }^{\circ} \mathrm{C}$ under $\mathrm{N}_{2}$ atmosphere. The mixture was then filtered with a $0.22 \mu \mathrm{m}$ PTFE membrane. The collected powder was extracted with $200 \mathrm{~mL}$ of boiling xylene for $48 \mathrm{~h}$ to remove un-grafted maleated $\mathrm{PE}$, and dried at $90{ }^{\circ} \mathrm{C}$ under vacuum overnight, thus immobilizing PE chains on the surface of MWNTs (PE- $g$-MWNTs).

\section{Characterization of $\mathrm{NH}_{2}$ terminated MWNTs and PE- $g$-MWNT}

X-ray Photon Scattering (XPS) scans were recorded on a Kratos Analytical instrument using Al monochromatic source (1.486 $\mathrm{keV}$ ) and the Fourier transform infrared (FTIR) spectra were recorded on a Thermo Nicolet 6700 in the wavelength range of $4000-400 \mathrm{~cm}^{-1}$ using $\mathrm{KBr}$ pellets. TGA was carried out under $\mathrm{N}_{2}$ atmosphere with a Pyris Diamond analyzer at a heating rate of $10{ }^{\circ} \mathrm{C} \min ^{-1}$. Transmission Electron Microscopy (TEM) images were acquired using Tecnai G2 F30 at $300 \mathrm{kV}$.

\section{Preparation of compatibilized blends}

Blends with and without compatibilizers (maleated $\mathrm{PE}$ ), and MWNTs (p-MWNT, $\mathrm{NH}_{2}$ terminated MWNTs and PE- $g$-MWNT) were prepared by melt mixing under $\mathrm{N}_{2}$ atmosphere in a laboratory scale twin screw extruder (Polylab, Thermohaake Minilab II) at $150{ }^{\circ} \mathrm{C}$ and $60 \mathrm{rpm}$ for $20 \mathrm{~min}$. The mini extruder has a recirculation channel which ensures complete mixing for desired time before the material can be taken out. Prior to melt mixing all the samples were vacuum dried overnight.

\section{Characterization of blends}

For tensile properties, dumbbell shaped specimens were prepared by compression molding at $150{ }^{\circ} \mathrm{C}$. The tensile tests were done on Instron Universal Testing Machine at room temperature with crosshead speed of $5 \mathrm{~mm} \mathrm{~min}^{-1}$. Morphological analysis for tensile fractured samples were assessed using field emission scanning electron microscopy (FE-SEM) on a ULTRA 55, FESEM, Carl Zesis with accelerating voltage of $5 \mathrm{kV}$. The morphology of the as pressed films were analyzed using SEM. Prior to SEM, the films were etched with cold sterilized distilled (DI) water to remove the PEO phase.

The melting temperature $\left(T_{\mathrm{m}}\right)$, crystallization temperature $\left(T_{\mathrm{c}}\right)$ and \% crystallinity $\left(X_{\mathrm{c}}\right)$ of $\mathrm{PE}$ (normalized with respect to weight and weight fraction) was measured using a Mettler Toledo DSC instrument with a heating and cooling rate of $10{ }^{\circ} \mathrm{C}$ $\min ^{-1}$. The degree of crystallinity of PE phase was calculated from the heat of fusion of second heating cycle. The heat of fusion $\left(\Delta H_{\mathrm{m}}\right)$ of $\mathrm{PE}$ phase was normalized to the fraction of polymer present in the blends. The degree of crystallinity $\left(X_{\mathrm{c}}\right)$ of $\mathrm{PE}$ phase was determined from the ratio of normalized heat 
of fusion ( $\Delta H_{\mathrm{m}}$, norm) to the heat of fusion of $100 \%$ crystalline $\mathrm{PE},\left(\Delta H_{\mathrm{f}}^{0}\right)$, which was taken as $293 \mathrm{~J} \mathrm{~g}^{-1} \cdot{ }^{9}$

\section{Results and discussion}

\section{Synthesis and characterization of $\mathrm{NH}_{2}$ terminated MWNTs and PE- $g$-MWNT}

As shown in Scheme 1, a two-step procedure was adopted to prepare $\mathrm{NH}_{2}$ terminated MWNTs. It consisted of $\mathrm{NH}_{2}$ attachment on to MWNT surface by acylation with thionyl chloride (SOCl) at $65{ }^{\circ} \mathrm{C}$ for $24 \mathrm{~h}$. This was followed by amidation of the resulted MWNT-COCl with an excess of EDA at $80{ }^{\circ} \mathrm{C}$ for $48 \mathrm{~h}$ to complete the functionalization process. The excess of EDA was then washed with anhydrous THF and filtered using $0.22 \mu \mathrm{m}$ PTFE membrane. In the second step, maleated PE was coupled to $\mathrm{NH}_{2}$ terminated MWNTs through the formation of amide groups, yielding PE- $g$-MWNTs, as shown in Scheme 1.

The direct evidence for EDA functionalized MWNTs and successful grafting of PE was provided by FTIR and XPS. Fig. 1a and $b$ illustrates the FTIR and XPS scans of $\mathrm{NH}_{2}$ terminated MWNTs and PE-g-MWNTs. A broad peak at $3424 \mathrm{~cm}^{-1}$ in the FTIR spectra indicates the presence of $(-\mathrm{OH})$ hydroxyl groups in MWNT-COOH. Upon coupling with EDA, a peak appears at $1653 \mathrm{~cm}^{-1}$ indicating the amide carbonyl $(\mathrm{C}=\mathrm{O})$ stretch. Similar observations were reported earlier. ${ }^{\mathbf{1 0 1 1}}$ The presence of $\mathrm{C}-\mathrm{N}$ stretching vibrations at $1044 \mathrm{~cm}^{-1}$ and the symmetric stretching at $3359 \mathrm{~cm}^{-1}$ confirms the presence of saturated primary amine on the MWNT surface. In the FTIR spectrum of PE- $g$-MWNTs, peaks at $1375 \mathrm{~cm}^{-1}$ and $1455 \mathrm{~cm}^{-1}$ are assigned to bending vibration of $\mathrm{CH}_{3}$, while peak at $1719 \mathrm{~cm}^{-1}$ indicates $\mathrm{C}=\mathrm{O}$ stretching vibration of maleic anhydride. In addition, peak at $2916 \mathrm{~cm}^{-1}$ shows stretching vibrations of PE. A shift of $\mathrm{C}=\mathrm{O}$ stretching vibration from 1709 to $1656 \mathrm{~cm}^{-1}$ confirms the covalent bonding of maleated PE on to MWNT surface. ${ }^{12,13}$

Functionalization of MWNTs with EDA is further confirmed by XPS. The presence of N1s peak in functionalized MWNTs indicates the presence of amine. Furthermore, N1s peak of $\mathrm{NH}_{2}$ terminated MWNTs was fitted to deconvolute the two species (see Fig. 1b). The lower binding energy (BE) peak at $396.9 \mathrm{eV}$ is attributed to terminal amine and the higher BE peak at $397.8 \mathrm{eV}$ could be attributed to the amide nitrogen. The latter showed

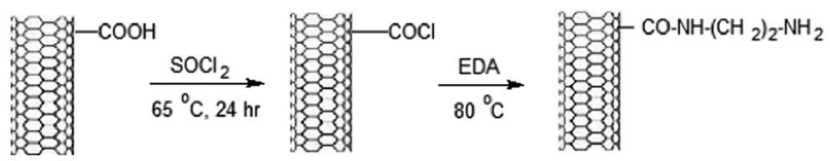

\section{$\mathrm{NH}_{2}$ terminated MWNTs}

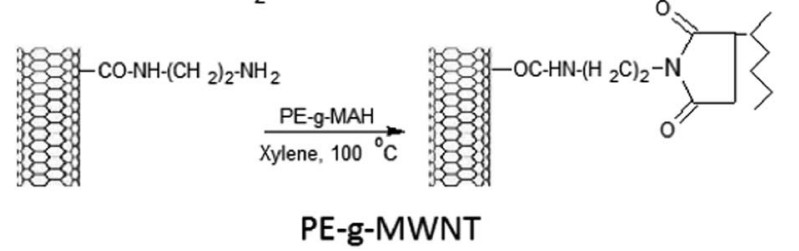

Scheme 1 Immobilizing PE on to MWNTs via reactive coupling of maleated PE with $\mathrm{NH}_{2}$ terminated MWNTs.
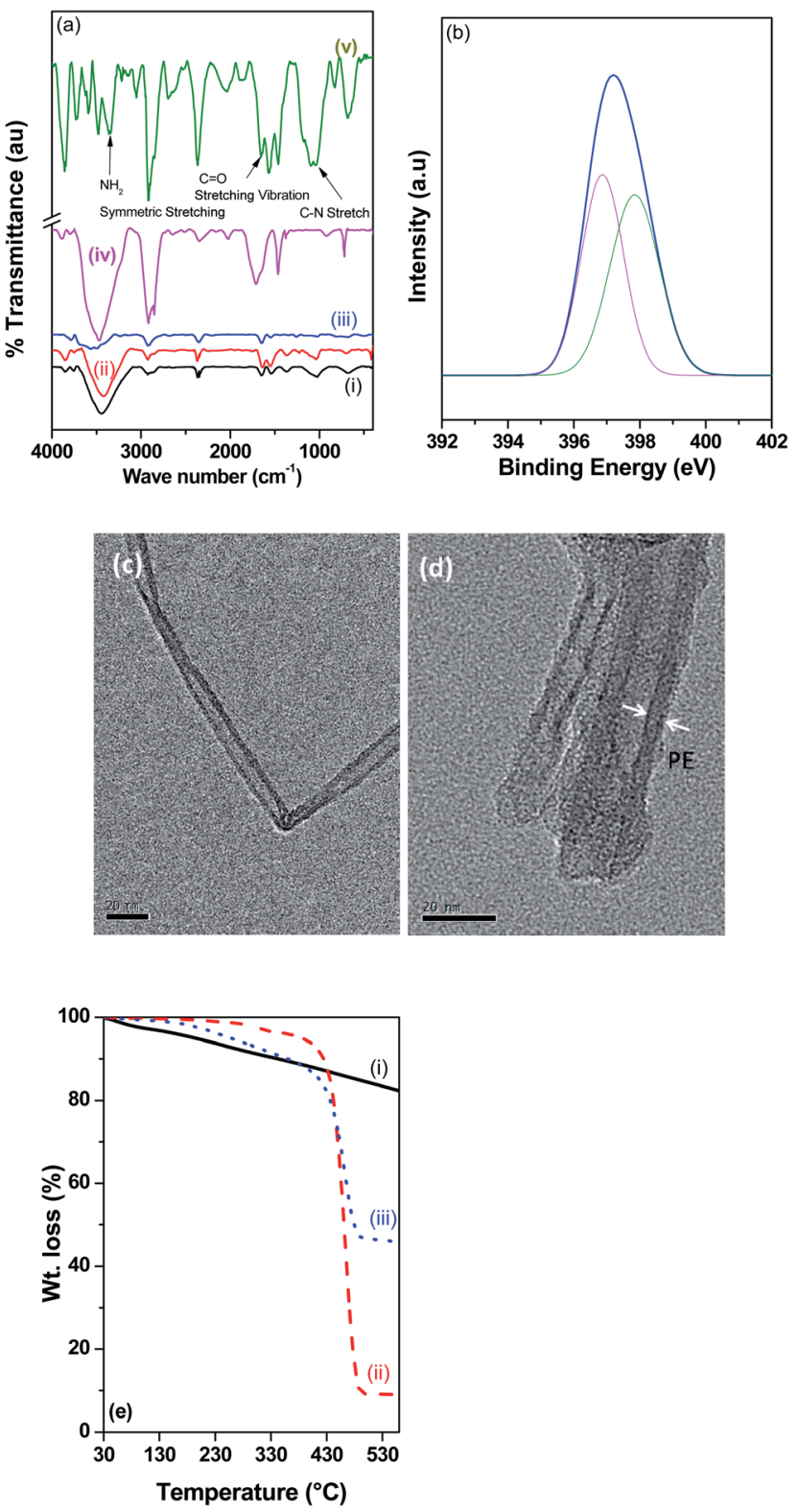

Fig. 1 (a) FT-IR spectra of (i) p-MWNTs, (ii) MWNT-COOH, (iii) $\mathrm{NH}_{2}$ terminated MWNTs, (iv) maleated PE and (v) PE-g-MWNT; (b) XPS scans of $\mathrm{NH}_{2}$ terminated MWNTs; TEM image of (c) MWNTs-COOH and (d) PE-g-MWNT (ex situ); (e) TGA thermogram of (i) neat $\mathrm{COOH}-$ MWNT (ii) maleated PE and (iii) PE-g-MWNT.

higher $\mathrm{BE}$ due to its electropositive nature and manifests the electron withdrawing nature of the carbonyl group. ${ }^{14,15}$

In general, immobilization of macromolecular chains on MWNTs can influence their thickness, which can be characterized by TEM and the fraction of polymer tethered on the MWNT surface can be evaluated by TGA. Fig. 1c-d shows the TEM micrographs of MWNT-COOH and PE-g-MWNTs. The latter showed a layer of polymer on the MWNTs as manifested from its increased diameter. This can result in improved dispersion of MWNTs in the PE phase of the blends. Fig. 1e shows the TGA scans for MWNT-COOH, maleated PE and PE- $g$ MWNT. At $550{ }^{\circ} \mathrm{C}$, the relative weight losses for MWNT-COOH, 
maleated PE and PE- $g$-MWNTs are 17\%, 91 and 54 wt $\%$ respectively. Large weight loss of PE- $g$-MWNTs is attributed to the thermal degradation of attached PE polymer chains. Thus confirming the maleated PE being grafted successfully on MWNTs. $^{13}$

\section{Structure-property relationship in PE- $g$-MWNTs compatibilized PE/PEO blends}

An efficient route to tailor the interface in binary polymer blends is by adding a compatibilizer. Next to the classical block copolymers, recent studies have revealed the key role of NPs in compatibilizing binary blends as discussed in the introduction section. In our earlier work, we have shown the potential of MWNTs in suppressing the coalescence of PMMA droplets in the PaMSAN matrix and the refinement of bi-continuous structures in PA6/ABS blends. Although, MWNTs render finer morphology irrespective of its localization in the blends, the mechanism is very different from the classical block copolymer route. It is understood from one of our previous work ${ }^{\mathbf{1 6}}$ that MWNTs localize in the thermodynamically less favored phase (PE) in PE/PEO blends which is driven by the flow characteristic of the components (the melt viscosity of PEO is significantly higher than PE). The key role of MWNTs as a compatibilizer may not be realized in PE/PEO blends due to lack of interfacial adhesion of MWNTs with the non-polar PE phase. Hence, in this study, we adopted two different approach to compatibilize PE/PEO blends: by in situ and ex situ immobilization of PE chains on to MWNTs as discussed before. However, both in situ and ex situ grafting of PE chains on to MWNTs can serve as an effective compatibilizer in PE/PEO blends. Recall, that the in situ grafting of $\mathrm{PE}$ chains on to MWNTs was realized by reactive coupling of maleated $\mathrm{PE}$ with $\mathrm{NH}_{2}$ terminated MWNTs during melt-mixing and the ex situ was realized by grafting maleated PE with $\mathrm{NH}_{2}$ terminated MWNTs a priori before melt mixing. Blends with pristine MWNT (p-MWNTs) was also prepared and investigated for their compatibilizing effects in PE/PEO blends.

The improved compatibility is revealed by SEM and is illustrated in Fig. 2a-g. To identify the minor phase, the specimens for SEM were immersed in DI water so that the dispersed PEO phase appears as holes in the PE matrix. Blends of PE/PEO are immiscible in nature and typically exhibit coarse morphology (see Fig. 2a). Clearly, the PEO holes in PE/PEO blends with different MWNTs (Fig. 2b-c) are much smaller than those of the neat blends, indicating that PEO is more finely in dispersed $\mathrm{PE} / \mathrm{PEO} / \mathrm{MWNT}$ blends. Interestingly, with addition of $3 \mathrm{wt} \%$ maleated PE in PE/PEO blends, the size of the dispersed PEO phase drastically reduced (see Fig. 2d and the inset of Fig. 2d) in striking contrast to the neat blends. This reduction in the PEO droplets clearly indicate the role of maleated PE in compatibilizing $\mathrm{PE} / \mathrm{PEO}$ blends by possibly reducing the interfacial tension between PE and PEO. ${ }^{17}$ More interestingly, the in situ grafting of maleated $\mathrm{PE}$ on to $\mathrm{NH}_{2}$ terminated MWNTs, through amidation during processing, has led to finer dispersion of PEO droplets in PE (see Fig. 2f) and which is not realized with p-MWNTs (see Fig. 2e). A schematic description of the compatibilizing mechanism of maleated $\mathrm{PE}$ and in situ grafting of $\mathrm{PE}$ on to MWNTs is displayed in Scheme 2. Due to high interfacial tension, macrophase separation exists in uncompatibilized PE/ PEO blends. With the introduction of maleated PE and PE- $g$ MWNTs, the interface is modified thereby reducing the unfavorable interactions. Consequently, the ex situ grafting of $\mathrm{PE}$ chains on to MWNTs led to better dispersion of MWNTs in the PE phase and the combination of maleated PE further resulted in a finer dispersion of PEO droplets in the blends (see Fig. 2g).

The reinforcing effect of MWNTs, maleated PE and PE- $g$ MWNTs is evaluated using tensile tests. Fig. 3 demonstrates the typical stress-strain curves for uncompatibilized and compatibilized by maleated PE and PE- $g$-MWNTs. Both PE and PEO show ductile behavior during uniaxial tensile test (not shown here). The control PEO exhibit a yield point followed by cold drawing and strain hardening (not shown here) whereas, the 70/30 PE/PEO blends exhibit a typical brittle failure with poor ultimate tensile strength and modulus. This is due to poor interfacial adhesion between PE/PEO. The corresponding tensile fracture morphology is shown in Fig. 4a. Clearly, the debonding at the interface is a cause of premature failure, thus lowering the mechanical properties with respect to the components (see Table 1). This is discussed in more details in the next section. A classical route to reduce the interfacial tension and improve the stress transfer at the interface is by compatibilizing. ${ }^{18}$ In the present work, we employed two different approaches; in situ and ex situ grafting of PE chains on to MWNTs. The use of maleated PE showed an increase of $109 \%$ in the ultimate tensile strength and 106\% in the Young's Modulus (YM) in the blends. This dramatic improvement in the mechanical properties is attributed to the specific interactions between maleic anhydride (MAH) and the terminal hydroxyl $(\mathrm{OH})$ groups in PEO. $^{7}$

Mechanical properties of the blends largely depend on the phase morphology and interfacial adhesion between the constituents. It is envisaged that the elongation at break is an important parameter to judge the interfacial adhesion between the phases, whereas the tensile strength is an indication of finer morphology, domain size and size homogeneity. ${ }^{19}$ Incorporation of p-MWNTs and $\mathrm{NH}_{2}$ terminated MWNTs showed an increase in YM by 55\% and $178 \%$ respectively; and in the ultimate tensile strength by $20 \%$ and $60 \%$ respectively. Although the MWNTs exhibit a reinforcement effect in the blends but the elongation at break is greatly sacrificed. This observation is a clear mandate to the fact that premature failure originates from the CNT aggregates ${ }^{19}$ and due to lack of interfacial adhesion with the matrix (here $\mathrm{PE}$ ) polymer. Interestingly, $\mathrm{NH}_{2}$ terminated MWNTs showed higher mechanical strength than the p-MWNT and can be attributed to possible interfacial interactions with PEO as well thereby acting as an interfacial modifier. In order to improve the interfacial adhesion and to tailor the interface, in situ grafting of maleated $\mathrm{PE}$ on to $\mathrm{NH}_{2}$ terminated MWNTs was facilitated during processing. The thus formed PE- $g$-MWNTs showed exceptionally high YM (186\%) and ultimate tensile strength (60\%) and more interestingly, retained the elongational properties. It is observed that for $1 \mathrm{wt} \%$ of PE- $g$ MWNT an increase of $78 \%$ in ultimate tensile strength and $143 \%$ in $\mathrm{YM}$ is recorded. 

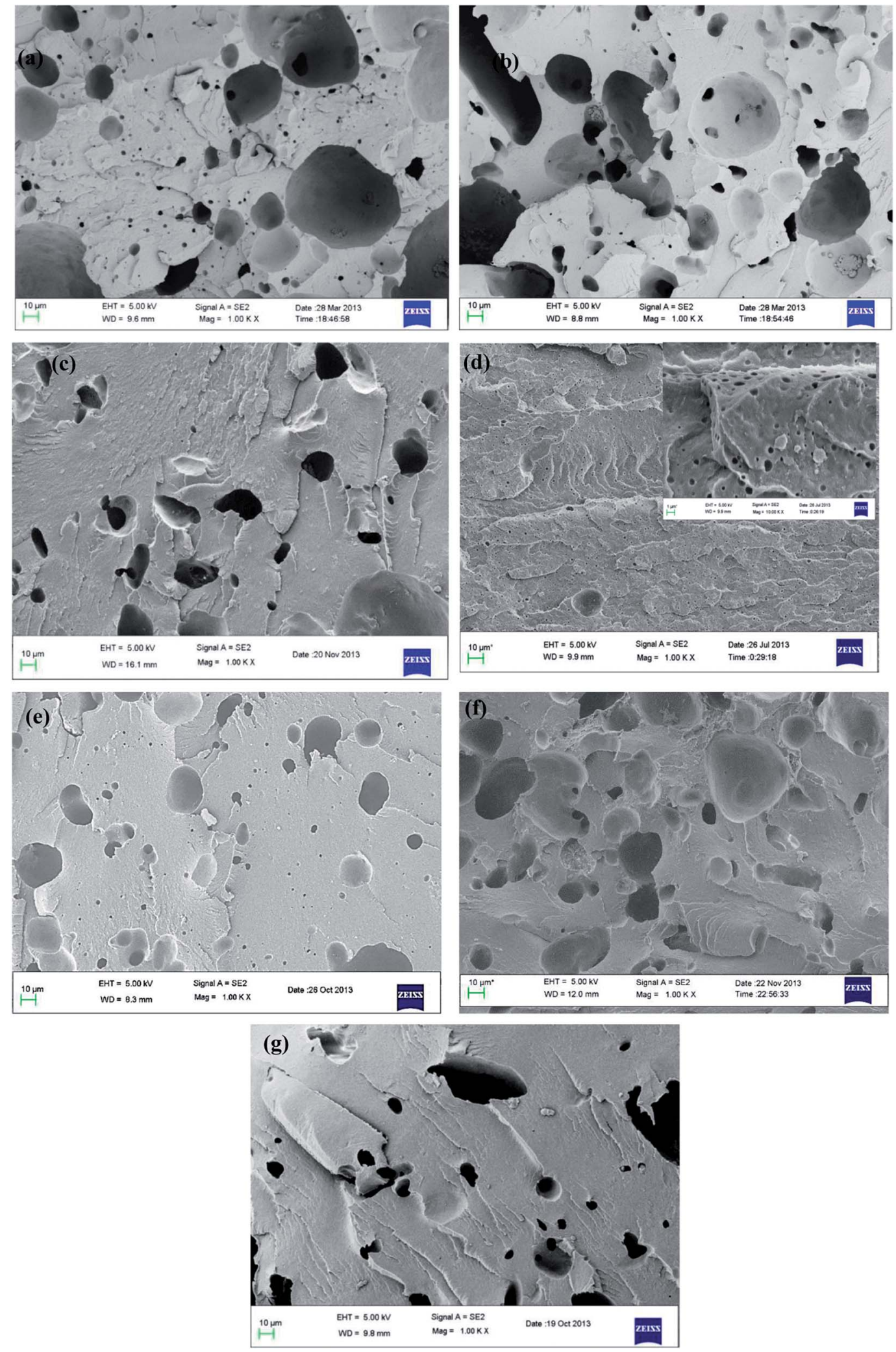

Fig. 2 Cryofractured morphology of (a) 70/30 PE/PEO blend (b) with 1 wt\% p-MWNTs (c) with 1 wt\% NH L $_{2}$ terminated MWNT (d) with 3 wt\% maleated PE (e) with 3 wt\% maleated PE and 1 wt $\%$ p-MWNTs (f) 3 wt\% maleated PE and with 1 wt $\%$ NH ${ }_{2}$ terminated MWNT (g) with 3 wt\% maleated PE and 1 wt\% PE-g-MWNTs. 

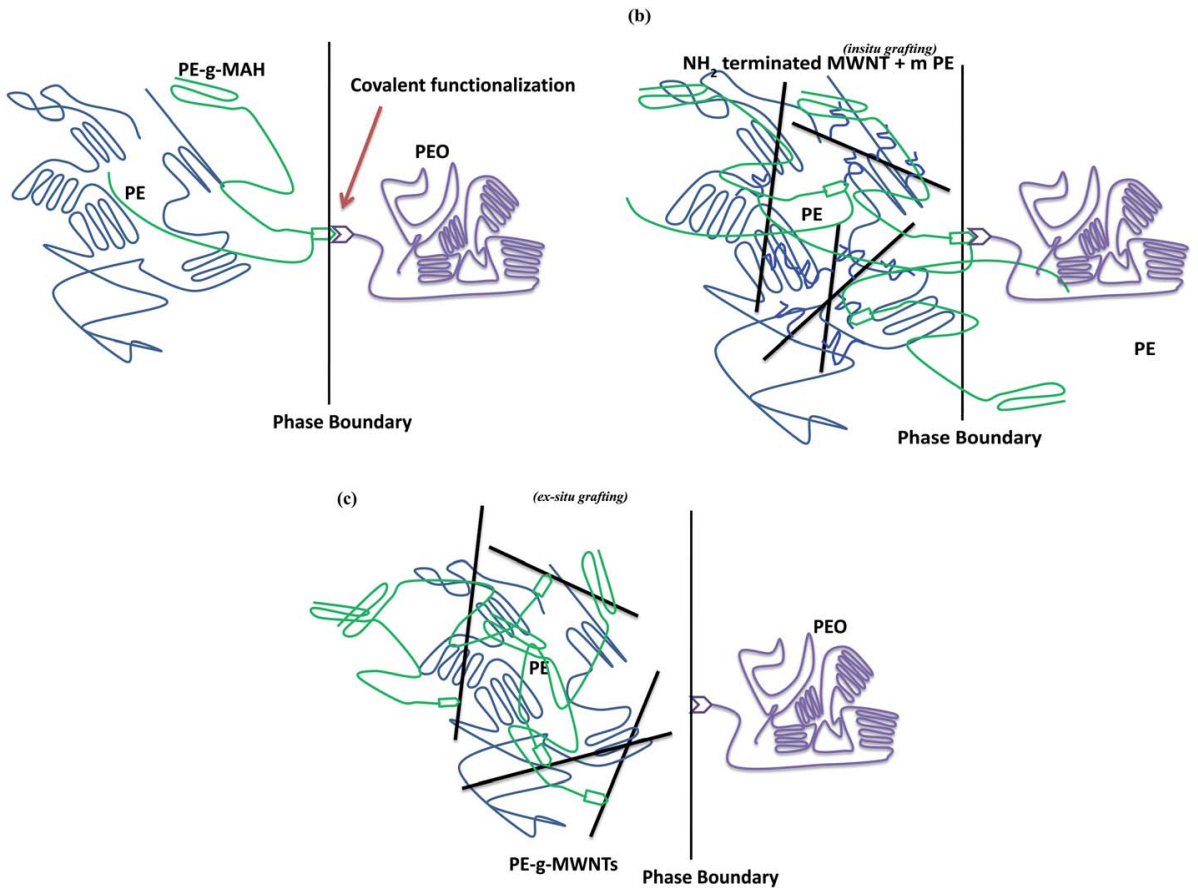

Scheme 2 A cartoon illustrating the various interactions of maleated PE and the modified MWNTs with the constituents (PE and PEO); (a) maleated PE (mPE) can interact with the hydroxyl end groups of PEO; (b) maleated PE can interact with $\mathrm{NH}_{2}$ terminated MWNTs in situ during melt-mixing and also with PEO (c) ex situ grafting of maleated $\mathrm{PE}$ with $\mathrm{NH}_{2}$ terminated MWNTs a priori before melt mixing.

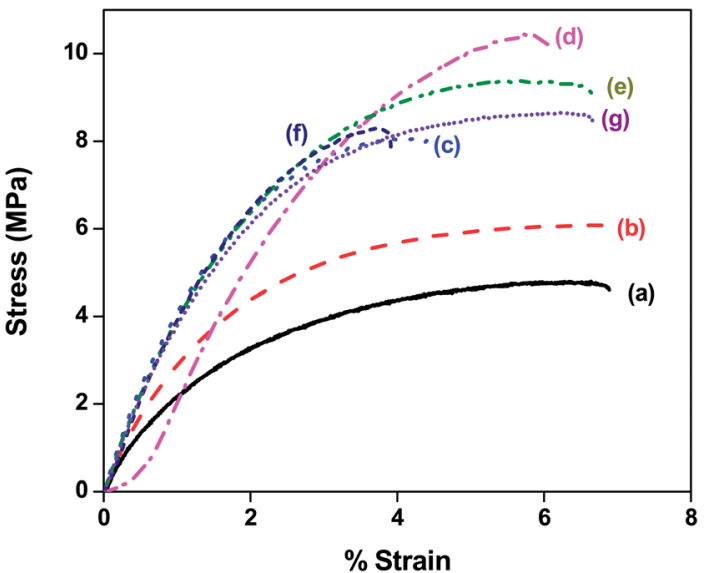

Fig. 3 Stress strain of (a) 70/30 PE/PEO blend (b) 1 wt\% p-MWNT (c) 1 wt $\% \mathrm{NH}_{2}$ terminated MWNT (d) with $3 \mathrm{wt} \%$ maleated PE, (e) with $3 \mathrm{wt} \%$ maleated PE and $1 \mathrm{wt} \% \mathrm{p}-\mathrm{MWNT}$, (f) with $3 \mathrm{wt} \%$ maleated PE and $1 \mathrm{wt} \%$ $\mathrm{NH}_{2}$ terminated MWNT and (g) with $3 \mathrm{wt} \%$ maleated PE and $1 \mathrm{wt} \% \mathrm{PE}-$ g-MWNTs.

In order to support the observations above and to understand the mechanism of fracture, the tensile fractured morphology is investigated in detail here and is shown in Fig. 4a-e. The tensile fractured surfaces of the neat 70/30 PE/ PEO blends reveal that PEO droplets is embedded in the PE matrix and with a weak interface. PE is a semicrystalline and ductile material, the lack of interfacial adhesion between PE and PEO caused an early failure with respect to the constituents. Peterlin $^{20}$ proposed that the component which has low strength and ductility (here PE), comprises of stacks of parallel lamellae with fewer links in-between which in turn deform plastically and spreads to maximum compliance before fracture. The typical failure in ductile semicrystalline polymer comprises of fibrillar morphology in the final stage of deformation. Hence, the fibrillar morphology observed in Fig. 4 b can be related with the fact that the PE phase shares the most load and due to poor interfacial adhesion, the stress transfer at the interface is relatively poor. Two interesting observations are noted here. Firstly, with the addition of maleated PE, the fibrils appear to be thinner with respect to the fractured surfaces of the neat blends manifesting the compatibilization rendered in the PE/PEO blends. Secondly, the fine dispersion of PEO phase in the PE matrix..$^{19,21}$

Incorporation of MWNTs in the blends led to reduction in the fibrillar morphology again suggesting poor interfacial adhesion between the phases. This observation is also reflected in the elongational properties of the blends. It is now understood that both fibrillar morphology and finer distribution of PEO phase are important to obtain improved mechanical properties in PE/PEO blends. In addition, MWNTs improve the YM of the blend significantly in striking contrast to the improvement in ultimate tensile strength. The latter property is strongly contingent on the interfacial adhesion between the phases; however, the reinforcement effected by MWNTs is well realized by the improvements in YM. A combination of MWNTs and maleated PE could result in simultaneous improvement in YM and ultimate tensile strength provided PE grafts are formed on the surface of MWNTs during melt mixing. In order to realize this, we compared the mechanical properties of the blends with 

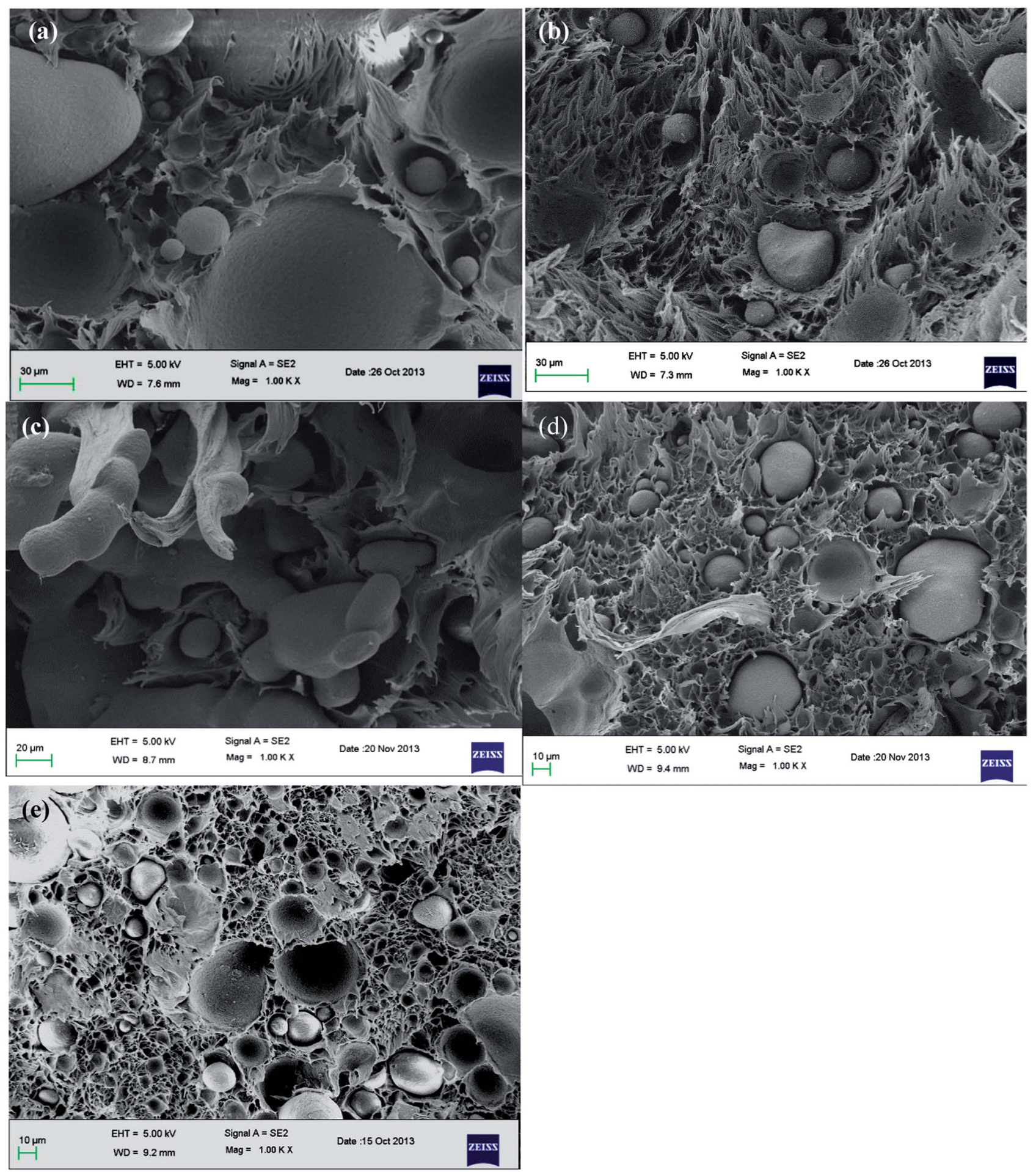

Fig. 4 Tensile fracture of (a) 70/30 PE/PEO blend (b) with 3 wt\% maleated PE (c) with 1 wt $\% \mathrm{NH}_{2}$ terminated MWNTs (d) 3 wt\% maleated PE and 1 wt\% $\mathrm{NH}_{2}$ terminated MWNTs (e) with 3 wt\% maleated PE and $1 \mathrm{wt} \% \mathrm{PE}-\mathrm{g}-\mathrm{MWNT}$.

ex situ PE-g-MWNTs with that of the in situ formed PE grafts on to MWNTs during processing. Interestingly, although ex situ formed PE-g-MWNTs are relatively well dispersed in the PE phase, the in situ formed PE-g-MWNTs resulted in significant improvement in YM and ultimate tensile strength. These observations are also supported by the tensile fractured morphology where finer dispersion of PEO phase is well evident next to improved interfacial adhesion, as seen in Fig. 5 where in situ formed grafts were observed to bridge the phases.

\section{Melting and crystallization behavior of the blends: effect of MWNTs}

Fig. 6 shows the cooling (Fig. 6a) and the heating scans (Fig. 6b) of $\mathrm{PE} / \mathrm{PEO}$ blends and the melting and crystallization 
Table 1 Mechanical properties of the 70/30 PE/PEO blend with compatibilizer and MWNTs

\begin{tabular}{|c|c|c|c|}
\hline Sample & $\begin{array}{l}\text { Ultimate } \\
\text { tensile } \\
\text { strength (MPa) }\end{array}$ & $\begin{array}{l}\text { Tensile } \\
\text { modulus } \\
(\mathrm{MPa})\end{array}$ & $\begin{array}{l}\text { Elongation } \\
\text { at } \\
\text { break (\%) }\end{array}$ \\
\hline Neat $70 / 30$ blend & $5 \pm 0.6$ & $173 \pm 8.0$ & $7.0 \pm 0.5$ \\
\hline With 1 wt\% p-MWNTs & $6 \pm 0.1$ & $268 \pm 7.6$ & $6.9 \pm 1.1$ \\
\hline $\begin{array}{l}\text { With } 1 \mathrm{wt} \% \mathrm{NH}_{2} \text {-t- } \\
\text { MWNTs }\end{array}$ & $8 \pm 0.1$ & $481 \pm 20$ & $4.4 \pm 0.3$ \\
\hline With 3 wt\% PE- $g$-MAH & $10 \pm 0.1$ & $356 \pm 4.6$ & $4.8 \pm 0.8$ \\
\hline $\begin{array}{l}\text { With } 3 \mathrm{wt} \% \text { PE- } g \text {-MAH } \\
\text { and } 1 \mathrm{wt} \% \text { p-MWNT }\end{array}$ & $9 \pm 0.6$ & $399 \pm 8.7$ & $6.0 \pm 0.8$ \\
\hline $\begin{array}{l}\text { With } 3 \mathrm{wt} \% \text { PE-g-MAH } \\
\text { and } 1 \mathrm{wt} \% \mathrm{NH}_{2} \text {-t-MWNTs }\end{array}$ & $8 \pm 0.2$ & $496 \pm 9.2$ & $3.9 \pm 0.5$ \\
\hline $\begin{array}{l}\text { With } 3 \text { wt } \% \text { PE- } g \text {-MAH } \\
\text { and } 1 \text { wt } \% \text { PE- } g \text {-MWNT }\end{array}$ & $8 \pm 0.2$ & $421 \pm 6.5$ & $6.5 \pm 1.1$ \\
\hline
\end{tabular}

temperatures for various blends investigated here are listed in Table 2. The corresponding exotherms of PE and PEO is well evident in the cooling scans of the blends; a typical characteristic of un-compatibilized blend. ${ }^{22}$ The crystallization temperatures $\left(T_{\mathrm{c}}\right)$ of PE and PEO are observed to be $100.4^{\circ} \mathrm{C}$ and $44.8^{\circ} \mathrm{C}$, respectively. Two interesting observations draw our attention. Firstly, with addition of $3 \mathrm{wt} \%$ maleated PE in PE/PEO blends, the $T_{\mathrm{c}}$ of PEO has been delayed by $c a .10{ }^{\circ} \mathrm{C}$ with respect to neat blends while the $T_{\mathrm{c}}$ of PE remains changed. Secondly, we observed fractionated crystallization in PE/PEO blends in the presence of maleated $\mathrm{PE}$. The melt interfacial reaction between maleated PE and the terminal hydroxyl group of PEO possibly has led to delayed crystallization in PEO. ${ }^{23}$ It is worth recalling that maleated PE has remarkably improved the dispersion of PEO in PE matrix (see Fig. 2d). It is envisaged that the heterogeneities are strongly contingent on the droplet size. For instance, the smaller droplets might contain less efficient heterogeneities and will only nucleate at the temperature when they become active. ${ }^{23} \mathrm{~A}$ moderate decrease in the melting temperature of PEO (see Fig. 6b) also suggests amorphousamorphous miscibility in the presence of maleated PE. In the presence of MWNTs, no appreciable change is observed in the crystallization temperature of either PE or PEO. It is envisaged that CNTs act as hetero nucleating agent and influence the rate of crystallization in semicrystalline polymer ${ }^{24,25}$ In this case, MWNTs or modified MWNTs did not show any appreciable change in the crystallization and melting temperature. Hence, it can be concluded that the observed changes in the structural properties are closely related with the strengthened interface on account of melt-interfacial interactions.
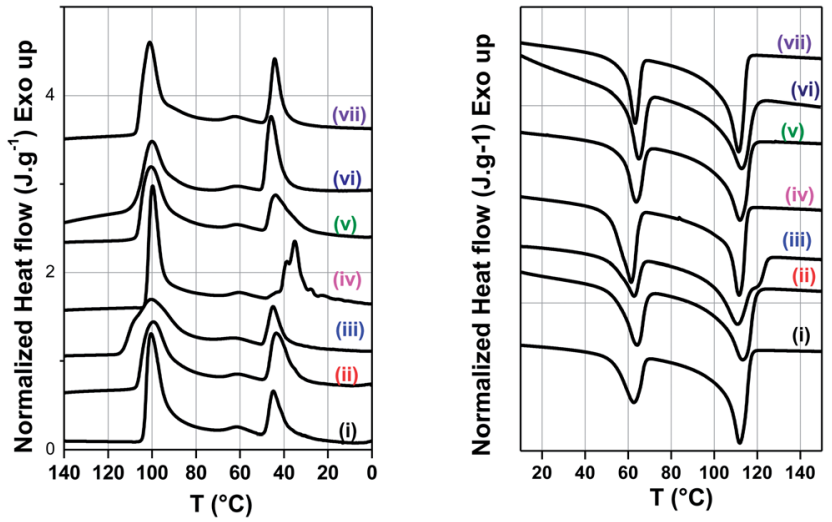

Fig. 6 DSC: (a) cooling and (b) second heating scans of blends of (i) 70/30 PE/PEO blend (ii) $1 \mathrm{wt} \% \mathrm{p}-\mathrm{MWNT}$ (iii) $1 \mathrm{wt} \% \mathrm{NH}_{2}$ terminated MWNT (iv) with 3 wt\% maleated PE, (v) with 3 wt\% maleated PE and 1 wt $\%$ p-MWNT, (vi) with 3 wt\% maleated PE and 1 wt $\% \mathrm{NH}_{2}$ terminated MWNT and (vii) with 3 wt\% maleated PE and 1 wt\% PE-g-MWNTs.

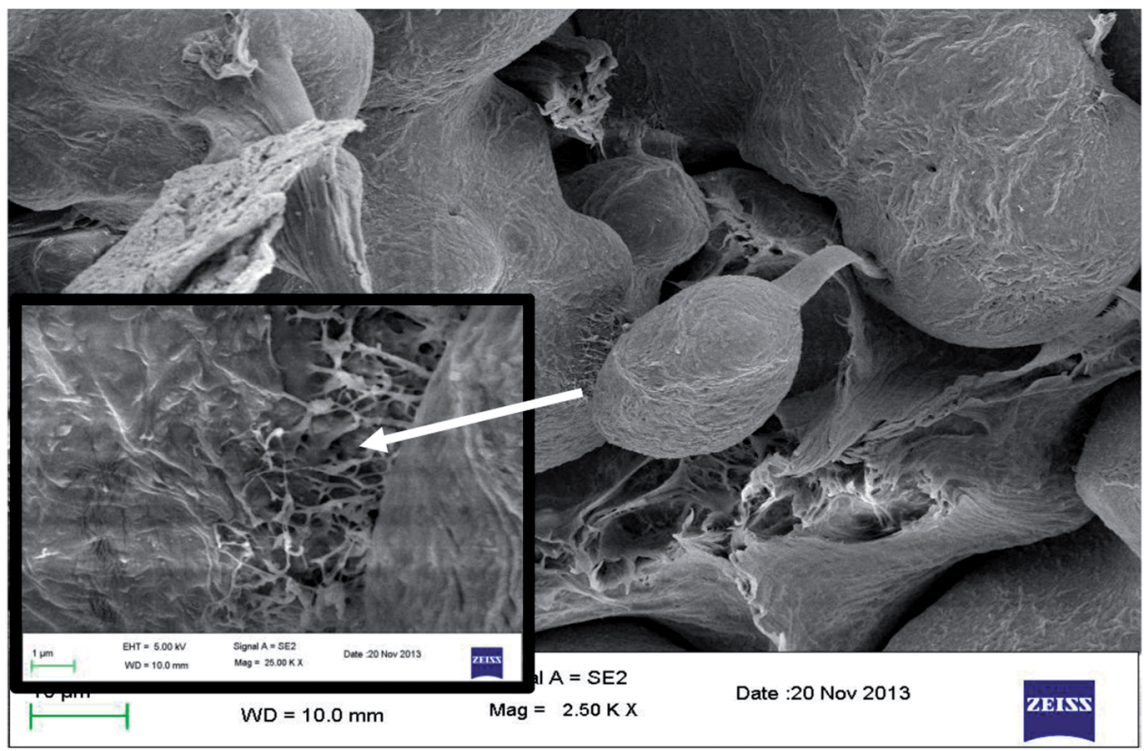

Fig. 5 High resolution SEM image of tensile fractured surface of 3 wt\% maleated PE and 1 wt $\% \mathrm{NH}_{2}$ terminated MWNTs. Inset shows MWNT bridging the phases. 
Table 2 DSC melting and crystallization parameter of the 70/30 PE/PEO blend with and without compatibilizer and MWNTs

\begin{tabular}{|c|c|c|c|c|}
\hline \multirow[b]{2}{*}{ Sample } & \multicolumn{2}{|c|}{$\begin{array}{l}\text { Melting temperature }\left(T_{\mathrm{m}}\right) \\
\left({ }^{\circ} \mathrm{C}\right)\end{array}$} & \multicolumn{2}{|c|}{$\begin{array}{l}\text { Crystallization temperature } \\
\left(T_{\mathrm{c}}\right)\left({ }^{\circ} \mathrm{C}\right)\end{array}$} \\
\hline & $\mathrm{PE}$ & PEO & $\mathrm{PE}$ & PEO \\
\hline Neat $70 / 30$ blend & 112 & 63 & 100 & 45 \\
\hline With 1 wt\% p-MWNTs & 114 & 64 & 100 & 44 \\
\hline With 1 wt $\% \mathrm{NH}_{2}$-t-MWNTs & 111 & 63 & 101 & 45 \\
\hline With 3 wt $\%$ PE- $g$-MAH & 111 & 61 & 100 & 35 \\
\hline With $3 \mathrm{wt} \%$ PE- $g$-MAH and $1 \mathrm{wt} \%$ p-MWNT & 112 & 64 & 101 & 44 \\
\hline With 3 wt $\%$ PE- $g$-MAH and 1 wt $\% \mathrm{NH}_{2}$-t-MWNTs & 113 & 65 & 100 & 46 \\
\hline With 3 wt $\%$ PE-g-MAH and 1 wt $\%$ PE-g-MWNT & 111 & 63 & 101 & 44 \\
\hline
\end{tabular}

\section{Conclusions}

In order to render finer morphology in the blends, various routes for compatibilization such as addition of maleated $\mathrm{PE}$ (PE grafted maleic anhydride), immobilizing PE chains, ex situ, onto MWNTs by covalent grafting, and in situ grafting of PE chains on to MWNTs during melt processing were adopted. Blends with modified MWNTs revealed a significant improvement in the mechanical properties of the blend besides remarkably improving the dispersion of the minor phase (PEO) in the blends. Blends with in situ formed PE-g-MWNTs showed exceptionally high Young's modulus (186\% higher with respect to neat blends) and ultimate tensile strength (60\% as compared to neat blends) and more interestingly, retained the elongational properties.

\section{Acknowledgements}

Department of Science and Technology (DSTO1150) and JATP is gratefully acknowledged for the financial support. We would like to thank MNCF, CeNSE for providing instrumentation facilities.

\section{References}

1 S. Bose, A. R. Bhattacharyya, A. R. Kulkarni and P. Pötschke, Electrical, rheological and morphological studies in cocontinuous blends of polyamide 6 and acrylonitrilebutadiene-styrene with multiwall carbon nanotubes prepared by melt blending, Compos. Sci. Technol., 2009, 69, 365-372.

2 L. A. Utracki, Polymer Blends Handbook, Kluwer Academic Pub, 2002.

3 J. R. Dygas, B. Misztal-Faraj, Z. Florjańczyk, F. Krok, M. Marzantowicz and E. Zygadło-Monikowska, Solid State Ionics, 2003, 157, 249.

4 O. Valentino, M. Sarno, N. G. Rainone, M. R. Nobile, P. Ciambelli, H. C. Neitzert and G. P. Simon, Influence of the polymer structure and nanotube concentration on the conductivity and rheological properties of polyethylene/ CNT composites, Phys. E, 2008, 40, 2440-2445.
5 M. Armand, J. M. Chabagno, M. Duclot, P. Vashista, J. N. Mundy and G. Shenoy, Fast Ion Transport in Solids, 1979. 6 S. A. Hashmi, A. Kumar, K. K. Maurya and S. Chandra, J. Phys. D: Appl. Phys., 1990, 23, 1307.

7 Y. L. Meltzer, Water-Soluble Polymers, Recent Developments, 1979.

8 S. D. Druger, A. Nitzan and M. A. Ratner, Phys. Rev. B: Condens. Matter Mater. Phys., 1985, 31, 3939.

9 S. Z. Cheng, Handbook of Thermal Analysis and Calorimetry: Applications to Polymers and Plastics, Elsevier, 2002.

10 B. K. Choi, Solid State Ionics, 2004, 168, 123.

11 K. C. Sobha and K. J. Rao, Solid State Ionics, 1995, 81, 145.

12 C. Chen, H. Pang, Z. Liu, Y.-B. Li, Y.-H. Chen, W.-Q. Zhang, X. Ji and J.-H. Tang, Enhanced foamability of isotactic polypropylene composites by polypropylene-graft-carbon nanotube, J. Appl. Polym. Sci., 2013, 130, 961-968.

13 C. Li, H. Deng, K. Wang, Q. Zhang, F. Chen and Q. Fu, Strengthening and toughening of thermoplastic polyolefin elastomer using polypropylene-grafted multiwalled carbon nanotubes, J. Appl. Polym. Sci., 2011, 121, 2104-2112.

14 B. X. Yang, K. P. Pramoda, G. Q. Xu and S. H. Goh, Mechanical Reinforcement of Polyethylene Using Polyethylene-Grafted Multiwalled Carbon Nanotubes, Adv. Funct. Mater., 2007, 17, 2062-2069.

15 B.-X. Yang, J.-H. Shi, K. P. Pramoda and S. H. Goh, Enhancement of the mechanical properties of polypropylene using polypropylene-grafted multiwalled carbon nanotubes, Compos. Sci. Technol., 2008, 68, 24902497.

16 P. K. Mural, G. Madras and S. Bose, Positive temperature coefficient and structural relaxations in selectively localized MWNTs in PE/PEO blends, RSC Adv., 2014, 4, 4943-4954.

17 J. Borah and T. Chaki, Dynamic rheological, morphology and mechanical properties of compatibilized LLDPE/EMA blends, J. Polym. Res., 2011, 18, 907-916.

18 A. Leclair and B. D. Favis, The role of interfacial contact in immiscible binary polymer blends and its influence on mechanical properties, Polymer, 1996, 37, 4723-4728.

19 S. Bose, A. R. Bhattacharyya, L. Häußler and P. Pötschke, Influence of multiwall carbon nanotubes on the mechanical properties and unusual crystallization behavior 
in melt-mixed co-continuous blends of polyamide6 and acrylonitrile butadiene styrene, Polym. Eng. Sci., 2009, 49, 1533-1543.

20 A. Peterlin, Molecular model of drawing polyethylene and polypropylene, J. Mater. Sci., 1971, 6, 490-508.

21 M. E. Villarreal, M. Tapia, S. M. Nuño-Donlucas, J. E. Puig and R. González-Núñez, Mechanical properties of polystyrene/polyamide 6 blends compatibilized with the ionomer poly(styrene-co-sodium acrylate), J. Appl. Polym. Sci., 2004, 92, 2545-2551.

22 H. Liu, T. Xie, Y. Zhang, Y. Ou and G. Yang, Crystallization behaviors of polypropylene/polyamide- 6 blends modified by a maleated thermoplastic elastomer, Polym. J., 2006, 38, 21-30.
23 C. Yordanov and L. Minkova, Fractionated crystallization of compatibilized LDPE/PA6 blends, Eur. Polym. J., 2005, 41, 527-534.

24 S. Bose, A. R. Bhattacharyya, P. V. Kodgire and A. Misra, Fractionated crystallization in PA6/ABS blends: Influence of a reactive compatibilizer and multiwall carbon nanotubes, Polymer, 2007, 48, 356-362.

25 S. Bose, A. R. Bhattacharyya, L. Häußler and P. Pötschke, Influence of multiwall carbon nanotubes on the mechanical properties and unusual crystallization behavior in melt-mixed co-continuous blends of polyamide6 and acrylonitrile butadiene styrene, Polym. Eng. Sci., 2009, 49, 1533-1543. 